\title{
THE SEARCH FOR NEW ANTIMICROBIAL AGENTS FROM THE SUBSTITUTED ARYLAMIDES OF 4-(4-OXO-4H-QUINAZOLIN- 3-YL)-PIPERIDINE-1-CARBOXYLIC ACIDS
}

\author{
K.Yu.Krolenko, S.V.Vlasov, I.O.Zhuravel, T.P.Osolodchenko*
}

National University of Pharmacy

53, Pushkinska str., Kharkiv, 61002, Ukraine. E-mail: krolenko.ky@gmail.com

*State Institution "Institute of Microbiology and Immunology named after I.I.Mechnikov of the National Academy of Medical Sciences of Ukraine"

Key words: quinazoline; piperidine; ureas; antimicrobials

The methods for the synthesis of the substituted arylamides of 4-(4-oxo-4H-quinazolin-3-yl)-piperidine-1-carboxylic acids using the technologies of the liquid-phase parallel synthesis have been developed. The structure of the compounds obtained has been confirmed by the data of the instrumental methods of organic analysis. The antibacterial activity of the compounds obtained has been studied using the agar "well" diffusion method against the standard test-strains of microorganisms. The results of the screening performed have shown that all compounds inhibit the growth of Staphylococcus aureus and Bacillus subtilis strains. The strains of Proteus vulgaris and Pseudomonas aeruginosa have been found to be the most resistant. The SAR-analysis of the substituted arylamides of 4-(4-oxo-4H-quinazolin-3-yl)-piperidine-1-carboxylic acids has demonstrated that the presence of the electron-donating substituents in position 8 of the quinazolin-4-one cycle and in position 4 of the aromatic fragment of the urea increases the activity of the compounds of this series against gram-positive bacteria. Such high efficacy of the lead compounds against the gram-positive bacterial strains can be applied for creating the narrow spectrum antibiotics derived from arylamides of 4-(4-oxo-4H-quinazolin-3-yl)-piperidine1-carboxylic acids.

\section{НОВІ АНТИМІКРОБНІ АГЕНТИ 3 РЯДУ ЗАМІЩЕНИХ АРИЛАМІДІВ 4-(4-ОКСО-4Н-ХІНАЗОЛІН-3-ІЛ)- ПІПЕРИДИН-1-КАРБОНОВИХ КИСЛОТ \\ К.Ю.Кроленко, С.В.Власов, І.О.Журавель, Т.П.Осолодченко}

Ключові слова: хіназолін; піперидин; сечовини; антимікробні засоби

Розроблені методики синтезу заміщених ариламідів 4-(4-оксо-4Н-хіназолін-3-іл)-піперидин-1-карбонових кислот з використанням технології рідиннофазного паралельного синтезу. Структуру отриманих сполук підтверджено даними фрізико-хімічних методів аналізу. Антибактеріальна активність синтезованих речовин досліджена методом «колодязів» із використанням стандартних тест-итамів мікроорганізмів. За результатами експерименту встановлено, що всі сполуки пригнічують picm Staphylococcus aureus ma Bacillus subtilis, а деякі значно перевищують активність препаратів порівняння. Найбільш стійкими до досліджуваних речовин виявились Proteus vulgaris ma Pseudomonas aeruginosa. Проведено SAR-аналіз для ряду отриманих заміщених ариламідів 4-(4-оксо-4Н-хіназолін-3-іл)-піперидин-1-карбонових кислот, згідно з яким встановлено, що наявність електронодонорних замісників у положенні 8 хіназолін-4-онового циклу та положенні 4 арильного фррагменту сечовини приводить до підвищення антибактеріальної активності даного ряду речовин по відношенню до грампозитивних бактерій. Наявність значної антимікробної активності сполук-лідерів по відношенню до грампозитивних итамів мікроорганізмів дозволяє говорити про перспективність створення антибактеріальних препаратів вузького спектра дії на основі заміщених ариламідів 4-(4-оксо-4Н-хіназолін-3-іл)-піперидин-1-карбонових кислот.

ПОИСК НОВЫХ АНТИМИКРОБНЫХ АГЕНТОВ ИЗ РЯДА ЗАМЕЩЕННЫХ АРИЛАМИДОВ 4-(4-ОКСО4Н-ХИНАЗОЛИН-3-ИЛ)-ПИПЕРИДИН-1-КАРБОНОВЫХ КИСЛОТ

К.Ю.Кроленко, С.В.Власов, И.А.Журавель, Т.П.Осолодченко

Ключевые слова: хиназолин; пиперидин; мочевины; противомикробные средства

Разработаны методики синтеза замещенных ариламидов 4-(4-оксо-4Н-хиназолин-3-ил)-пиперидин-1карбоновых кислот с использованием технологии жидкофразного параллельного синтеза. Структуру полученных соединений подтверждено данными фризико-химических методов анализа. Антибактериальная активность синтезированных веществ исследована методом «колодиев» с использованием стандартных тест-итаммов микроорганизмов. По результатам эксперимента установлено, что все соединения угнетают рост Staphylococcus aureus u Bacillus subtilis, а некоторые значительно превышают активность препаратов сравнения. Наиболее стойкими к исследуемым веществам оказались Proteus vulgaris u Pseudomonas aeruginosa. Проведен SAR-анализ для ряда полученных замещенных ариламидов 4-(4-оксо-4Н-хиназолин-3-ил)-пиперидин-1-карбоновых кислот, согласно которого установлено, что наличие электронодонорных заместителей в положении 8 хиназолин-4-онового цикла и положении 4 арильного фрагмента мочевины приводит к повышению антибактериальной активности данного ряда соединений по отношению к грамположительным бактериям. Наличие значительной антимикробной активности соединений-лидеров по отношению к грамположительным штаммам микроорганизмов позволяет говорить о перспективности создания антибактериальных препаратов узкого спектра действия на основе замещенных ариламидов 4-(4-оксо-4Н-хиназолин-3-ил)-пиперидин-1-карбоновых кислот. 
The reported information of the last years confirms that substituted arylamides 4-piperidinyl-1-carboxylic acid are known as antibacterial [1, 2] and antituberculosis agents $[3,4]$. The compounds of the similar structure may be useful for treatment and prevention of prostate cancer, cancer of the gastrointestinal tract [5]; they were also proposed as Tamoxifen analogues [6]. The antagonistic activity for CRTH2 receptors makes these compounds appropriate for asthma therapy [7].

The derivatives of quinazolin-4-one modified in position 3 with the 4-piperidyl fragment, in their turn, possess the antiviral activity against Flaviviridae species, which are the ethological factor of hepatitis $\mathrm{C}$ (HCV) [8].

Considering the wide spectrum of the physiological activity of the compounds bearing the fragments of either arylamides 4-piperidinyl-1-carboxylic acid or the moiety of 3-(piperidin-4-yl)quinazolin-4(3H)-one the combination of these two pharmacophore fragments have chosen as the way for the new promising biologically active compounds. They were also tested for the antimicrobial activity.

The synthesis of 3-(piperidin-4-yl)quinazolin-4(3H)ones 1 was performed in two steps by cyclization of 4-piperidylamides of 2-aminobenzoic acid protected with urethane with triethyl orthoformate at the first step, and further hydrolysis of the esters obtained as it was reported previously [9]. To achieve the wide chemical diversity for the heterocyclic compounds with the secondary nitrogen atom the acylation with derivatives of carboxylic acid [10], isocyanates [11], and sulphonyl chlorides $[9,12]$ together with the alkylation reaction [13] is commonly applied.

Earlier we successfully carried out the interaction of compounds $\mathbf{1}$ with aryl sulphonyl chlorides [9], resulting in the series of sulphonylanides with the antibacterial activity against gram-positive and gramnegative bacteria and some fungi. The interaction products of amines $\mathbf{1}$ with carboxylic acid imidazolides and their alkylation with benzyl chlorides and chloroacetoamides are the compounds with rather low melting points, which complicate their isolation and increase the cost of their synthesis for the purposes of medicinal chemistry and screening studies. That is why the reaction of amines $\mathbf{1}$ with isocyanates has been cho- sen as the better way for obtaining the wide diversity of the promising biologically active compounds suitable for screening studies.

The reaction conditions for interaction of $\mathbf{1}$ with isocyanates were improved in few steps. First, it was found that when heating in DMF a great amount of bis-urea was formed probably because of the self interaction of isocyanate; application of 1,4-dioxane required precipitation of the product by dilution with water and its further crystallization. It is known that the interaction of isocyanates with alcohols results in urethanes [14], but regardless of this fact 2-propanol has been chosen as a suitable solvent for this interaction. The main reason for the choice is a perfect 2-propanol solubility of the staring amine $\mathbf{1}$ and the poor solubility of ureas $\mathbf{2}$. It is interesting that even traces of urethanes were not identified as impurities for ureas 2 obtained. To minimize the possibility of the by-product formation the reaction was performed at ambient temperature. Using the technologies of the parallel liquid phase synthesis compounds $\mathbf{1}$ were reacted with aryl isocyanates in 2-propanol, it resulted in the series of the corresponding ureas $\mathbf{2}$ with the substituents of the different electronic structure in the aromatic ring (Scheme).

The data for compounds (2.1-2.25) obtained is presented in Table 1; their ${ }^{1} \mathrm{H}$ NMR data are given in Table 1, 2.

All ${ }^{1} \mathrm{H}$ NMR-spectra of compounds 2.1-2.25 contain the signals of methylene groups of the piperidine cycle at 1.80-2.17 та 4.22-4.33 ppm; the hydrogen atom in position 4 of the piperidine cycle gives the resonance peak at 4.80-4.90 ppm as a multiplet. For all compounds 2 the singlet proton signal in position 2 of the quinazoline cycle is observed at $8.40-8.50 \mathrm{ppm}$. The aromatic protons of this aromatic moiety resonate in the region of $6.90-7.80 \mathrm{ppm}$. The signals of amide aromatic protons CONHAr as to their multiplicity are typical for the corresponding substituted phenyl radicals for each specific case.

The antibacterial activity of the compounds obtained was studied using the agar "well" diffusion method against the standard test-strains of gram-positive and gram-negative bacteria and fungi according to the international standards $[15,16]$. The results of the screening are presented in the Tab. 3.

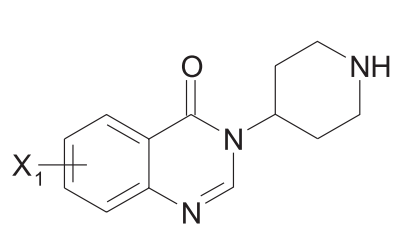

1.1-1.7

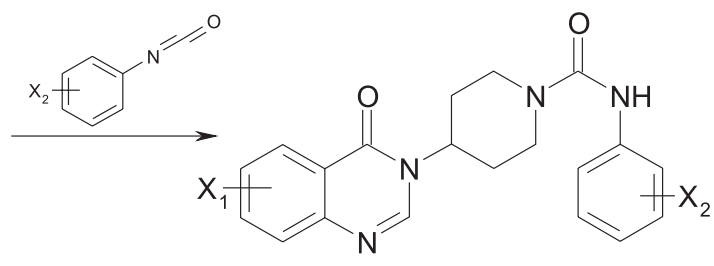

2.1-2.25

1.1 $\mathrm{X}_{1}=8-\mathrm{Me} ; 1.2 \mathrm{X}_{1}=8-\mathrm{Cl} ; 1.3 \mathrm{X}_{1}=7-\mathrm{F} ; 1.4 \mathrm{X}_{1}=6-\mathrm{Cl} ; 1.5 \mathrm{X}_{1}=6-\mathrm{F} ; 1.6 \mathrm{X}_{1}=\mathrm{H} ; 1.7 \mathrm{X}_{1}=7-\mathrm{Cl}$

Scheme. The synthesis of the substituted arylamides of 4-(4-oxo-4H-quinazolin-3-yl)-piperidine-1-carboxylic acids 2. 
Table 1

Arylamides of 4-(4-oxo-4H-quinazolin-3-yl)-piperidine-1-carboxylic acids 2

\begin{tabular}{|c|c|c|c|c|c|c|}
\hline No. & $X_{1}$ & $\mathrm{X}_{2}$ & $\begin{array}{c}\text { Molecular formula, } \\
\text { M. m. }\end{array}$ & $\begin{array}{c}\mathrm{N}, \% \\
\text { calc./exp. }\end{array}$ & M. p., ${ }^{\circ} \mathrm{C}$ & Yield, \% \\
\hline 2.1 & 8-Me & 2-OMe, 5-Cl & $\begin{array}{c}\mathrm{C}_{22} \mathrm{H}_{23} \mathrm{ClN}_{4} \mathrm{O}_{3} \\
426.90\end{array}$ & $\frac{13.12}{13.37}$ & $189-191$ & 77 \\
\hline 2.2 & 8-Me & $\mathrm{H}$ & $\begin{array}{c}\mathrm{C}_{21} \mathrm{H}_{22} \mathrm{~N}_{4} \mathrm{O}_{2} \\
362.42\end{array}$ & $\frac{15.46}{15.80}$ & 208-210 & 76 \\
\hline 2.3 & 8-Me & 3,4-ethylendioxy & $\begin{array}{c}\mathrm{C}_{23} \mathrm{H}_{24} \mathrm{~N}_{4} \mathrm{O}_{4} \\
420.46\end{array}$ & $\frac{13.33}{12.99}$ & $227-229$ & 79 \\
\hline 2.4 & 8-Me & 2,4-diF & $\begin{array}{c}\mathrm{C}_{21} \mathrm{H}_{20} \mathrm{~F}_{2} \mathrm{~N}_{4} \mathrm{O}_{2} \\
398.41\end{array}$ & $\frac{14.06}{14.32}$ & $217-219$ & 80 \\
\hline 2.5 & 8-Me & 2-OMe, 5-Me & $\begin{array}{c}\mathrm{C}_{23} \mathrm{H}_{26} \mathrm{~N}_{4} \mathrm{O}_{3} \\
406.48\end{array}$ & $\frac{13.78}{14.05}$ & $173-175$ & 75 \\
\hline 2.6 & 8-Me & 3,5-diMe & $\begin{array}{c}\mathrm{C}_{23} \mathrm{H}_{26} \mathrm{~N}_{4} \mathrm{O}_{2} \\
390.48\end{array}$ & $\frac{14.35}{14.58}$ & $230-232$ & 77 \\
\hline 2.7 & 8-Me & 4-OEt & $\begin{array}{c}\mathrm{C}_{23} \mathrm{H}_{26} \mathrm{~N}_{4} \mathrm{O}_{3} \\
406.48\end{array}$ & $\frac{13.78}{13.51}$ & $219-221$ & 78 \\
\hline 2.8 & 8-Me & 2-OMe & $\begin{array}{l}\mathrm{C}_{22} \mathrm{H}_{24} \mathrm{~N}_{4} \mathrm{O}_{3} \\
392.45\end{array}$ & $\frac{14.28}{13.98}$ & $185-187$ & 80 \\
\hline 2.9 & 8-Me & 2-Me, 3-Cl & $\begin{array}{c}\mathrm{C}_{22} \mathrm{H}_{23} \mathrm{ClN}_{4} \mathrm{O}_{2} \\
410.90\end{array}$ & $\frac{13.64}{13.43}$ & $216-218$ & 79 \\
\hline 2.10 & 8-Me & 3-OMe & $\begin{array}{c}\mathrm{C}_{22} \mathrm{H}_{24} \mathrm{~N}_{4} \mathrm{O}_{3} \\
392.45\end{array}$ & $\frac{14.28}{14.06}$ & $202-204$ & 76 \\
\hline 2.11 & 8-Me & 4-OMe & $\begin{array}{c}\mathrm{C}_{22} \mathrm{H}_{24} \mathrm{~N}_{4} \mathrm{O}_{3} \\
392.45\end{array}$ & $\frac{14.28}{14.53}$ & |194-196 & 75 \\
\hline 2.12 & $8-\mathrm{Cl}$ & $4-E t$ & $\begin{array}{c}\mathrm{C}_{22} \mathrm{H}_{23} \mathrm{ClN}_{4} \mathrm{O}_{2} \\
410.90\end{array}$ & $\frac{13.64}{13.41}$ & $210-212$ & 78 \\
\hline 2.13 & $8-\mathrm{Cl}$ & 3-F, 4-Me & $\begin{array}{c}\mathrm{C}_{21} \mathrm{H}_{20} \mathrm{ClFN}_{4} \mathrm{O}_{2} \\
414.86\end{array}$ & $\frac{13.50}{13.62}$ & $239-241$ & 80 \\
\hline 2.14 & $7-\mathrm{Cl}$ & $4-E t$ & $\begin{array}{c}\mathrm{C}_{22} \mathrm{H}_{23} \mathrm{CIN}_{4} \mathrm{O}_{2} \\
410.90\end{array}$ & $\frac{13.64}{13.38}$ & $261-263$ & 79 \\
\hline 2.15 & $7-\mathrm{Cl}$ & 3,4-diMe & $\begin{array}{c}\mathrm{C}_{22} \mathrm{H}_{23} \mathrm{ClN}_{4} \mathrm{O}_{2} \\
410.90\end{array}$ & $\frac{13.64}{13.82}$ & $240-242$ & 76 \\
\hline 2.16 & $7-\mathrm{Cl}$ & 4-Me & $\begin{array}{c}\mathrm{C}_{21} \mathrm{H}_{21} \mathrm{ClN}_{4} \mathrm{O}_{2} \\
396.87\end{array}$ & $\frac{14.12}{13.86}$ & $237-239$ & 77 \\
\hline 2.17 & $7-\mathrm{F}$ & 2-OEt & $\begin{array}{c}\mathrm{C}_{22} \mathrm{H}_{23} \mathrm{FN}_{4} \mathrm{O}_{3} \\
410.44\end{array}$ & $\frac{13.65}{13.41}$ & $170-172$ & 75 \\
\hline 2.18 & $7-\mathrm{F}$ & 2-OMe, 5-Me & $\begin{array}{c}\mathrm{C}_{22} \mathrm{H}_{23} \mathrm{FN}_{4} \mathrm{O}_{3} \\
410.44\end{array}$ & $\frac{13.65}{13.85}$ & $218-220$ & 79 \\
\hline 2.19 & $7-\mathrm{F}$ & 2,4-diMe & $\begin{array}{l}\mathrm{C}_{22} \mathrm{H}_{23} \mathrm{FN}_{4} \mathrm{O}_{2} \\
394.44\end{array}$ & $\frac{14.20}{13.98}$ & $252-254$ & 80 \\
\hline 2.20 & $7-\mathrm{F}$ & 2-Me, 5-Cl & $\begin{array}{c}\mathrm{C}_{21} \mathrm{H}_{20} \mathrm{ClFN}_{4} \mathrm{O}_{2} \\
414.86\end{array}$ & $\frac{13.50}{13.73}$ & 194-196 & 76 \\
\hline 2.21 & $6-\mathrm{Cl}$ & 3-OMe & $\begin{array}{l}\mathrm{C}_{21} \mathrm{H}_{21} \mathrm{ClN}_{4} \mathrm{O}_{3} \\
412.87\end{array}$ & $\frac{13.57}{13.39}$ & $230-232$ & 76 \\
\hline 2.22 & $6-\mathrm{Cl}$ & 2-OEt & $\begin{array}{c}\mathrm{C}_{22} \mathrm{H}_{23} \mathrm{ClN}_{4} \mathrm{O}_{3} \\
426.90\end{array}$ & $\frac{13.12}{12.92}$ & $206-208$ & 78 \\
\hline 2.23 & $6-\mathrm{Cl}$ & 3,4-ethylendioxy & $\begin{array}{c}\mathrm{C}_{22} \mathrm{H}_{21} \mathrm{ClN}_{4} \mathrm{O}_{4} \\
440.88\end{array}$ & $\frac{12.71}{12.97}$ & $242-244$ & 80 \\
\hline 2.24 & $6-\mathrm{F}$ & 2-OMe, 5-Cl & $\begin{array}{c}\mathrm{C}_{21} \mathrm{H}_{20} \mathrm{ClFN}_{4} \mathrm{O}_{3} \\
430.86\end{array}$ & $\frac{13.00}{13.12}$ & $236-238$ & 75 \\
\hline 2.25 & $\mathrm{H}$ & 4-Me & $\begin{array}{c}\mathrm{C}_{21} \mathrm{H}_{22} \mathrm{~N}_{4} \mathrm{O}_{2} \\
362.42\end{array}$ & $\frac{15.46}{15.18}$ & $225-227$ & 77 \\
\hline
\end{tabular}


The 1H NMR spectral data of arylamides of 4-(4-oxo-4H-quinazolin-3-yl)-piperidine-1-carboxylic acids 2

\begin{tabular}{|c|c|c|}
\hline \multirow{2}{*}{ No. } & \multicolumn{2}{|c|}{ Chemical shift, $\delta$, ppm. } \\
\hline & Aliphatic protons & Aromatic protons, $\mathrm{NH}$ \\
\hline 1 & 2 & 3 \\
\hline 2.1 & \begin{tabular}{|l} 
1.81-2.10 (4H, m, 3,5- $\left.\mathrm{CH}_{2}\right) ; 2.53\left(3 \mathrm{H}, \mathrm{s}, 8-\mathrm{CH}_{3}\right) ;$ \\
3.81 (3H, s, $\left.2^{\prime}-\mathrm{OCH}_{3}\right) ; 2.90-3.10(2 \mathrm{H}, \mathrm{m})$ and \\
$4.11-4.32\left(2 \mathrm{H}, \mathrm{m}, 2,6-\mathrm{CH}_{2}\right) ; 4.72-4.93(1 \mathrm{H}, \mathrm{m}, 4-\mathrm{CH})$
\end{tabular} & $\begin{array}{l}\text { 7.01 (2H, s) and 7.75-7.90 (2H, m, 3,',',6'-Ar, NH); } 7.42(1 \mathrm{H}, \\
\text { t, H-6 Ar); } 7.67(1 \mathrm{H}, \mathrm{d}, \mathrm{H}-7 \mathrm{Ar}) ; 8.05(1 \mathrm{H}, \mathrm{d}, \mathrm{H}-5 \mathrm{Ar}) ; 8.49 \\
(1 \mathrm{H}, \mathrm{s}, \mathrm{H}-2 \mathrm{Ar})\end{array}$ \\
\hline 2.2 & $\begin{array}{l}\text { 1.80-2.13 }\left(4 \mathrm{H}, \mathrm{m}, 3,5-\mathrm{CH}_{2}\right) ; 2.54\left(3 \mathrm{H}, \mathrm{s}, 8-\mathrm{CH}_{3}\right) ; \\
\text { 2.82-3.07 }(2 \mathrm{H}, \mathrm{m}) \text { and 4.26-4.45 }\left(2 \mathrm{H}, \mathrm{m}, 2,6-\mathrm{CH}_{2}\right) ; \\
\text { 4.71-4.93 }(1 \mathrm{H}, \mathrm{m}, 4-\mathrm{CH})\end{array}$ & $\begin{array}{l}6.91\left(1 \mathrm{H}, \mathrm{t}, \mathrm{H}-4^{\prime} \mathrm{Ar}\right) ; 7.23\left(2 \mathrm{H}, \mathrm{t}, \mathrm{H}-3^{\prime}, 5^{\prime} \mathrm{Ar}\right) ; 7.35-7.55(3 \mathrm{H}, \mathrm{m}, \\
\left.\mathrm{H}-6,2^{\prime}, 6^{\prime} \mathrm{Ar}\right) ; 7.67(1 \mathrm{H}, \mathrm{d}, \mathrm{H}-7 \mathrm{Ar}) ; 8.03(1 \mathrm{H}, \mathrm{d}, \mathrm{H}-5 \mathrm{Ar}) ; 8.48 \\
(1 \mathrm{H}, \mathrm{s}, \mathrm{H}-2 \mathrm{Ar}) ; 8.53(1 \mathrm{H}, \mathrm{s}, \mathrm{NH})\end{array}$ \\
\hline 2.3 & $\begin{array}{l}\text { 1.78-2.17 }\left(4 \mathrm{H}, \mathrm{m}, 3,5-\mathrm{CH}_{2}\right) ; 2.53\left(3 \mathrm{H}, \mathrm{s}, 8-\mathrm{CH}_{3}\right) ; \\
\text { 2.81-3.01 }(2 \mathrm{H}, \mathrm{m}) \text { and 4.22-4.41 }\left(2 \mathrm{H}, \mathrm{m}, 2,6-\mathrm{CH}_{2}\right) ; \\
4.15\left(4 \mathrm{H}, \mathrm{s}, 2,3 "-\mathrm{CH}_{2}\right) ; 4.72-4.95(1 \mathrm{H}, \mathrm{m}, 4-\mathrm{CH})\end{array}$ & $\begin{array}{l}\text { 6.63-7.15 (3H, m, H-2,'5,'6 Ar); } 7.42(1 \mathrm{H}, \mathrm{t}, \mathrm{H}-6 \mathrm{Ar}) ; \\
\text { 7.68 (1H, d, H-7 Ar); } 8.02(1 \mathrm{H}, \mathrm{d}, \mathrm{H}-5 \mathrm{Ar}) ; \\
8.48(1 \mathrm{H}, \mathrm{s}, \mathrm{H}-2 \mathrm{Ar}) ; 8.51(1 \mathrm{H}, \mathrm{s}, \mathrm{NH})\end{array}$ \\
\hline 2.4 & $\begin{array}{l}\text { 1.79-2.12 }\left(4 \mathrm{H}, \mathrm{m}, 3,5-\mathrm{CH}_{2}\right) ; 2.52\left(3 \mathrm{H}, \mathrm{s}, 8-\mathrm{CH}_{3}\right) ; \\
\text { 2.89-3.08 }(2 \mathrm{H}, \mathrm{m}) \text { and 4.22-4.41 }\left(2 \mathrm{H}, \mathrm{m}, 2,6-\mathrm{CH}_{2}\right) ; \\
\text { 4.72-4.93 }(1 \mathrm{H}, \mathrm{m}, 4-\mathrm{CH})\end{array}$ & $\begin{array}{l}\text { 6.93-7.47 (4H, m, H-6,3'5,'6 Ar); } 7.67(1 \mathrm{H}, \mathrm{d}, \mathrm{H}-7 \mathrm{Ar}) ; \\
8.01(1 \mathrm{H}, \mathrm{d}, \mathrm{H}-5 \mathrm{Ar}) ; 8.38(1 \mathrm{H}, \mathrm{s}, \mathrm{NH}) ; 8.49(1 \mathrm{H}, \mathrm{s}, \mathrm{H}-2 \mathrm{Ar})\end{array}$ \\
\hline 2.5 & $\begin{array}{l}\text { 1.80-2.11 }\left(4 \mathrm{H}, \mathrm{m}, 3,5-\mathrm{CH}_{2}\right) ; 2.17\left(3 \mathrm{H}, \mathrm{s}, 5^{\prime}-\mathrm{CH}_{3}\right) ; \\
\text { 2.51 }\left(3 \mathrm{H}, \mathrm{s}, 8-\mathrm{CH}_{3}\right) ; 3.78\left(3 \mathrm{H}, \mathrm{s}, 2^{\prime}-\mathrm{OCH}_{3}\right) ; \\
\text { 2.85-3.05 }(2 \mathrm{H}, \mathrm{m}) \text { and 4.15-4.35 }\left(2 \mathrm{H}, \mathrm{m}, 2,6-\mathrm{CH}_{2}\right) ; \\
\text { 4.71-4.93 }(1 \mathrm{H}, \mathrm{m}, 4-\mathrm{CH})\end{array}$ & $\begin{array}{l}\text { 6.75-6.90 (2H, m) and 7.62-7.73 (2H, m, H-7, 3,', Ar, NH); } \\
7.42(1 \mathrm{H}, \mathrm{t}, \mathrm{H}-6 \mathrm{Ar}) ; 7.51\left(1 \mathrm{H}, \mathrm{s}, \mathrm{H}-6^{\prime} \mathrm{Ar}\right) ; 8.01 \\
(1 \mathrm{H}, \mathrm{d}, \mathrm{H}-5 \mathrm{Ar}) ; 8.50(1 \mathrm{H}, \mathrm{s}, \mathrm{H}-2 \mathrm{Ar})\end{array}$ \\
\hline 2.6 & $\begin{array}{l}1.80-2.12\left(4 \mathrm{H}, \mathrm{m}, 3,5-\mathrm{CH}_{2}\right) ; 2.19\left(6 \mathrm{H}, \mathrm{s}, 3^{\prime}, 5^{\prime}-\mathrm{CH}_{3}\right) ; \\
2.52\left(3 \mathrm{H}, \mathrm{s}, 8-\mathrm{CH}_{3}\right) ; 2.83-3.00(2 \mathrm{H}, \mathrm{m}) \text { and } \\
4.21-4.38\left(2 \mathrm{H}, \mathrm{m}, 2,6-\mathrm{CH}_{2}\right) ; 4.70-4.93(1 \mathrm{H}, \mathrm{m}, 4-\mathrm{CH})\end{array}$ & $\begin{array}{l}6.58\left(1 \mathrm{H}, \mathrm{s}, \mathrm{H}-4^{\prime} \mathrm{Ar}\right) ; 7.09\left(2 \mathrm{H}, \mathrm{s}, \mathrm{H}-2{ }^{\prime} 6^{\prime} \mathrm{Ar}\right) ; 7.40(1 \mathrm{H}, \mathrm{t}, \mathrm{H}-6 \\
\mathrm{Ar}) ; 7.65(1 \mathrm{H}, \mathrm{d}, \mathrm{H}-7 \mathrm{Ar}) ; 8.01(1 \mathrm{H}, \mathrm{d}, \mathrm{H}-5 \mathrm{Ar}) ; \\
8.43(1 \mathrm{H}, \mathrm{s}, \mathrm{NH}) ; 8.51(1 \mathrm{H}, \mathrm{s}, \mathrm{H}-2 \mathrm{Ar})\end{array}$ \\
\hline 2.7 & $\begin{array}{l}1.35\left(3 \mathrm{H}, \mathrm{t}, \mathrm{CH}_{3}\right), 1.82-2.10\left(4 \mathrm{H}, \mathrm{m}, 3,5-\mathrm{CH}_{2}\right) ; 2.55(3 \mathrm{H}, \\
\left.\mathrm{s}, 8-\mathrm{CH}_{3}\right) ; 3.96\left(2 \mathrm{H}, \mathrm{q}, \mathrm{CH}_{2}\right) ; 2.88-2.99(2 \mathrm{H}, \mathrm{m}) \text { and } \\
4.31-4.43\left(2 \mathrm{H}, \mathrm{m}, 2,6-\mathrm{CH}_{2}\right) ; 4.78-4.93(1 \mathrm{H}, \mathrm{m}, 4-\mathrm{CH})\end{array}$ & $\begin{array}{l}6.74\left(2 \mathrm{H}, \mathrm{d}, \mathrm{H}-3^{\prime}, 5^{\prime} \mathrm{Ar}\right) ; 7.34\left(2 \mathrm{H}, \mathrm{d}, \mathrm{H}-2^{\prime}, 6^{\prime} \mathrm{Ar}\right) ; 7.40(1 \mathrm{H}, \mathrm{t}, \\
\mathrm{H}-6 \mathrm{Ar}) ; 7.62(1 \mathrm{H}, \mathrm{d}, \mathrm{H}-7 \mathrm{Ar}) ; 8.03(1 \mathrm{H}, \mathrm{d}, \mathrm{H}-5 \mathrm{Ar}) ; \\
8.34(1 \mathrm{H}, \mathrm{s}, \mathrm{NH}) ; 8.45(1 \mathrm{H}, \mathrm{s}, \mathrm{H}-2 \mathrm{Ar})\end{array}$ \\
\hline 2.8 & $\begin{array}{l}\text { 1.80-2.15 }\left(4 \mathrm{H}, \mathrm{m}, 3,5-\mathrm{CH}_{2}\right) ; 2.52\left(3 \mathrm{H}, \mathrm{s}, 8-\mathrm{CH}_{3}\right) ; \\
\text { 3.81 }\left(3 \mathrm{H}, \mathrm{s}, 2^{\prime}-\mathrm{OCH}_{3}\right) ; 2.85-3.04(2 \mathrm{H}, \mathrm{m}) \text { and } \\
4.18-4.35\left(2 \mathrm{H}, \mathrm{m}, 2,6-\mathrm{CH}_{2}\right) ; 4.71-4.93(1 \mathrm{H}, \mathrm{m}, 4-\mathrm{CH})\end{array}$ & $\begin{array}{l}\text { 6.70-7.05 (3H, m) and 7.60-7.75 (3H, m, H-7, 3,', ', 5,', } \mathrm{6}^{\prime} \mathrm{Ar}, \\
\mathrm{NH}) ; 7.41(1 \mathrm{H}, \mathrm{t}, \mathrm{H}-6 \mathrm{Ar}) ; 8.01(1 \mathrm{H}, \mathrm{d}, \mathrm{H}-5 \mathrm{Ar}) ; 8.49 \\
(1 \mathrm{H}, \mathrm{s}, \mathrm{H}-2 \mathrm{Ar})\end{array}$ \\
\hline 2.9 & \begin{tabular}{|l|}
$1.82-2.12\left(4 \mathrm{H}, \mathrm{m}, 3,5-\mathrm{CH}_{2}\right) ; 2.20\left(3 \mathrm{H}, \mathrm{s}, \mathrm{2}^{\prime}-\mathrm{CH}_{3}\right) ;$ \\
2.55 (3H, s, $\left.8-\mathrm{CH}_{3}\right) ; 2.85-3.05(2 \mathrm{H}, \mathrm{m})$ and \\
$4.22-4.38\left(2 \mathrm{H}, \mathrm{m}, 2,6-\mathrm{CH}_{2}\right) ; 4.73-4.93(1 \mathrm{H}, \mathrm{m}, 4-\mathrm{CH})$
\end{tabular} & $\begin{array}{l}\text { 7.10-7.26 (3H, m, H-4,'5,6 Ar); } 7.42(1 \mathrm{H}, \mathrm{t}, \mathrm{H}-6 \mathrm{Ar}) \\
\text { 7.67 (1H, d, H-7 Ar); } 8.01(1 \mathrm{H}, \mathrm{d}, \mathrm{H}-5 \mathrm{Ar}) ; 8.37(1 \mathrm{H}, \mathrm{s}, \mathrm{NH}) ; \\
8.50(1 \mathrm{H}, \mathrm{s}, \mathrm{H}-2 \mathrm{Ar})\end{array}$ \\
\hline 2.10 & $\begin{array}{l}\text { 1.80-2.16 }\left(4 \mathrm{H}, \mathrm{m}, 3,5-\mathrm{CH}_{2}\right) ; 2.54\left(3 \mathrm{H}, \mathrm{s}, 8-\mathrm{CH}_{3}\right) ; \\
\text { 2.85-3.05 }(2 \mathrm{H}, \mathrm{m}) \text { and 4.28-4.32 }\left(2 \mathrm{H}, \mathrm{m}, 2,6-\mathrm{CH}_{2}\right) ; \\
3.69\left(3 \mathrm{H}, \mathrm{s}, 3^{\prime}-\mathrm{OCH}_{3}\right) ; 4.70-4.90(1 \mathrm{H}, \mathrm{m}, 4-\mathrm{CH})\end{array}$ & $\begin{array}{l}\text { 6.45-6.54 }(1 \mathrm{H}, \mathrm{m}) \text { and 7.03-7.20 (3H, m, H-2,',',5,6 Ar); } \\
\text { 7.42 (1H, t, H-6 Ar); 7.67 (1H, d, H-7 Ar); } 8.00(1 \mathrm{H}, \mathrm{d} \\
\mathrm{H}-5 \mathrm{Ar}) ; 8.48(1 \mathrm{H}, \mathrm{s}, \mathrm{H}-2 \mathrm{Ar}) ; 8.56(1 \mathrm{H}, \mathrm{s}, \mathrm{NH})\end{array}$ \\
\hline 2.11 & $\begin{array}{l}\text { 1.80-2.15 }\left(4 \mathrm{H}, \mathrm{m}, 3,5-\mathrm{CH}_{2}\right) ; 2.55\left(3 \mathrm{H}, \mathrm{s}, 8-\mathrm{CH}_{3}\right) ; \\
\left.\text { 2.80-3.02 }(2 \mathrm{H}, \mathrm{m}) \text { and 4.23-4.42(2H, m, 2,6-CH }{ }_{2}\right) ; \\
3.71\left(3 \mathrm{H}, \mathrm{s}, 4^{\prime}-\mathrm{OCH}_{3}\right) ; 4.71-4.92(1 \mathrm{H}, \mathrm{m}, 4-\mathrm{CH})\end{array}$ & $\begin{array}{l}6.72\left(2 \mathrm{H}, \mathrm{d}, \mathrm{H}-3^{\prime}, 5^{\prime} \mathrm{Ar}\right) ; 7.35\left(2 \mathrm{H}, \mathrm{d}, \mathrm{H}-2^{\prime}, 6^{\prime} \mathrm{Ar}\right) ; 7.48(1 \mathrm{H}, \mathrm{t}, \\
\mathrm{H}-6 \mathrm{Ar}) ; 7.68 \mathrm{(1H}, \mathrm{d}, \mathrm{H}-7 \mathrm{Ar}) ; 8.02(1 \mathrm{H}, \mathrm{d}, \mathrm{H}-5 \mathrm{Ar}) ; \\
8.43(1 \mathrm{H}, \mathrm{s}, \mathrm{NH}) ; 8.53(1 \mathrm{H}, \mathrm{s}, \mathrm{H}-2 \mathrm{Ar})\end{array}$ \\
\hline 2.12 & $\begin{array}{l}1.18\left(3 \mathrm{H}, \mathrm{t}, \mathrm{CH}_{3}\right), 1.83-2.15\left(4 \mathrm{H}, \mathrm{m}, 3,5-\mathrm{CH}_{2}\right) ; \\
\text { 2.56 }\left(2 \mathrm{H}, \mathrm{q}, \mathrm{CH}_{2}\right) ; 2.83-3.00(2 \mathrm{H}, \mathrm{m}) \text { and } \\
4.35-4.46\left(2 \mathrm{H}, \mathrm{m}, 2,6-\mathrm{CH}_{2}\right) ; 4.76-4.90(1 \mathrm{H}, \mathrm{m}, 4-\mathrm{CH})\end{array}$ & $\begin{array}{l}7.03\left(2 \mathrm{H}, \mathrm{d}, \mathrm{H}-3^{\prime}, 5^{\prime} \mathrm{Ar}\right) ; 7.35\left(2 \mathrm{H}, \mathrm{d}, \mathrm{H}-2^{\prime}, 6^{\prime} \mathrm{Ar}\right) ; 7.49(1 \mathrm{H}, \mathrm{t}, \\
\mathrm{H}-6 \mathrm{Ar}) ; 7.92(1 \mathrm{H}, \mathrm{d}, \mathrm{H}-7 \mathrm{Ar}) ; 8.13(1 \mathrm{H}, \mathrm{d}, \mathrm{H}-5 \mathrm{Ar}) ; \\
8.43(1 \mathrm{H}, \mathrm{s}, \mathrm{NH}) ; 8.57(1 \mathrm{H}, \mathrm{s}, \mathrm{H}-2 \mathrm{Ar})\end{array}$ \\
\hline 2.13 & $\begin{array}{l}\text { 1.81-2.13 }\left(4 \mathrm{H}, \mathrm{m}, 3,5-\mathrm{CH}_{2}\right) ; 2.17\left(3 \mathrm{H}, \mathrm{s}, 4^{\prime}-\mathrm{CH}_{3}\right) ; \\
\text { 2.90-3.00 }(2 \mathrm{H}, \mathrm{m}) \text { and 4.31-4.45 }\left(2 \mathrm{H}, \mathrm{m}, 2,6-\mathrm{CH}_{2}\right) ; \\
\text { 4.78-4.90(1H, m, 4-CH) }\end{array}$ & $\begin{array}{l}7.04(1 \mathrm{H}, \mathrm{m}) \text { and } 7.14(1 \mathrm{H}, \mathrm{m}) \text { and } 7.38\left(1 \mathrm{H}, \mathrm{m}, \mathrm{H}-2^{\prime}, 5^{\prime}, 6 \mathrm{Ar}\right) ; \\
7.49(1 \mathrm{H}, \mathrm{t}, \mathrm{H}-6 \mathrm{Ar}) ; 7.92(1 \mathrm{H}, \mathrm{d}, \mathrm{H}-7 \mathrm{Ar}) ; 8.13(1 \mathrm{H}, \mathrm{d}, \mathrm{H}-5 \\
\mathrm{Ar}) ; 8.57(1 \mathrm{H}, \mathrm{s}, \mathrm{H}-2 \mathrm{Ar}) ; 8.62(1 \mathrm{H}, \mathrm{s}, \mathrm{NH})\end{array}$ \\
\hline 2.14 & $\begin{array}{l}1.13\left(3 \mathrm{H}, \mathrm{t}, \mathrm{CH}_{3}\right) ; 1.78-2.17\left(4 \mathrm{H}, \mathrm{m}, 3,5-\mathrm{CH}_{2}\right) ; \\
2.51\left(2 \mathrm{H}, \mathrm{q}, \mathrm{CH}_{2}\right) ; 2.78-3.04(2 \mathrm{H}, \mathrm{m}) \text { and } \\
4.21-4.43\left(2 \mathrm{H}, \mathrm{m}, 2,6-\mathrm{CH}_{2}\right) ; 4.69-4.91(1 \mathrm{H}, \mathrm{m}, 4-\mathrm{CH})\end{array}$ & $\begin{array}{l}7.04\left(2 \mathrm{H}, \mathrm{d}, \mathrm{H}-3^{\prime}, 5^{\prime} \mathrm{Ar}\right) ; 7.36\left(2 \mathrm{H}, \mathrm{d}, \mathrm{H}-2^{\prime} \mathrm{6}^{\prime} \mathrm{Ar}\right) ; 7.56(1 \mathrm{H}, \mathrm{dd} \\
\mathrm{H}-6 \mathrm{Ar}) ; 7.74(1 \mathrm{H}, \mathrm{d}, \mathrm{H}-8 \mathrm{Ar}) ; 8.14(1 \mathrm{H}, \mathrm{d}, \mathrm{H}-5 \mathrm{Ar}) ; \\
8.47(1 \mathrm{H}, \mathrm{s}, \mathrm{NH}) ; 8.53(1 \mathrm{H}, \mathrm{s}, \mathrm{H}-2 \mathrm{Ar})\end{array}$ \\
\hline 2.15 & $\begin{array}{l}\text { 1.79-2.11 }\left(4 \mathrm{H}, \mathrm{m}, 3,5-\mathrm{CH}_{2}\right) ; 2.14\left(6 \mathrm{H}, \mathrm{s}, 3^{\prime} \mathrm{4}^{\prime}-\mathrm{CH}_{3}\right) ; \\
\text { 2.72-3.02 }(2 \mathrm{H}, \mathrm{m}) \text { and 4.21-4.42 }\left(2 \mathrm{H}, \mathrm{m}, 2,6-\mathrm{CH}_{2}\right) ; \\
\text { 4.69-4.91 }(1 \mathrm{H}, \mathrm{m}, 4-\mathrm{CH})\end{array}$ & $\begin{array}{l}6.96\left(1 \mathrm{H}, \mathrm{m}, \mathrm{H}-2^{\prime} \mathrm{Ar}\right) ; 7.11-7.28\left(2 \mathrm{H}, \mathrm{m}, \mathrm{H}-5^{\prime}, 6^{\prime} \mathrm{Ar}\right) ; \\
7.56(1 \mathrm{H}, \mathrm{d}, \mathrm{H}-6 \mathrm{Ar}) ; 7.74(1 \mathrm{H}, \mathrm{s}, \mathrm{H}-8 \mathrm{Ar}) ; 8.13(1 \mathrm{H}, \mathrm{d}, \mathrm{H}-5 \\
\mathrm{Ar}) ; 8.37(1 \mathrm{H}, \mathrm{s}, \mathrm{NH}) ; 8.53(1 \mathrm{H}, \mathrm{s}, \mathrm{H}-2 \mathrm{Ar})\end{array}$ \\
\hline 2.16 & $\begin{array}{l}\text { 1.77-2.13 }\left(4 \mathrm{H}, \mathrm{m}, 3,5-\mathrm{CH}_{2}\right) ; 2.21\left(3 \mathrm{H}, \mathrm{s}, 4^{\prime}-\mathrm{CH}_{3}\right) ; \\
\text { 2.72-3.00 }(2 \mathrm{H}, \mathrm{m}) \text { and 4.21-4.45 }\left(2 \mathrm{H}, \mathrm{m}, 2,6-\mathrm{CH}_{2}\right) ; \\
\text { 4.65-4.91 }(1 \mathrm{H}, \mathrm{m}, 4-\mathrm{CH})\end{array}$ & $\begin{array}{l}7.02\left(2 \mathrm{H}, \mathrm{d}, \mathrm{H}-3^{\prime}, 5^{\prime} \mathrm{Ar}\right) ; 7.35\left(2 \mathrm{H}, \mathrm{d}, \mathrm{H}-2^{\prime} \mathbf{6}^{\prime} \mathrm{Ar}\right) ; 7.56(1 \mathrm{H}, \mathrm{d} \\
\mathrm{H}-6 \mathrm{Ar}) ; 7.72(1 \mathrm{H}, \mathrm{s}, \mathrm{H}-8 \mathrm{Ar}) ; 8.15(1 \mathrm{H}, \mathrm{d}, \mathrm{H}-5 \mathrm{Ar}) ; \\
8.47(1 \mathrm{H}, \mathrm{s}, \mathrm{NH}) ; 8.57(1 \mathrm{H}, \mathrm{s}, \mathrm{H}-2 \mathrm{Ar})\end{array}$ \\
\hline 2.17 & $\begin{array}{l}1.41\left(3 \mathrm{H}, \mathrm{t}, \mathrm{CH}_{3}\right) ; 1.81-2.18\left(4 \mathrm{H}, \mathrm{m}, 3,5-\mathrm{CH}_{2}\right) ; \\
\text { 2.96-3.08 }(2 \mathrm{H}, \mathrm{m}) \text { and } 4.21-4.35\left(2 \mathrm{H}, \mathrm{m}, 2,6-\mathrm{CH}_{2}\right) ; \\
4.09\left(2 \mathrm{H}, \mathrm{q}, \mathrm{CH}_{2}\right) ; 4.77-4.92(1 \mathrm{H}, \mathrm{m}, 4-\mathrm{CH})\end{array}$ & $\begin{array}{l}\text { 6.81-6.90 (1H, m, H-5' Ar); 6.92-6.98 (2H, m) and 7.31-7.42 } \\
\left(2 \mathrm{H}, \mathrm{m}, \mathrm{H}-5,3^{\prime}, 4^{\prime} \mathbf{6}^{\prime} \mathrm{Ar}\right) ; 7.57(1 \mathrm{H}, \mathrm{s}, \mathrm{NH}) ; 7.77(1 \mathrm{H}, \mathrm{d}, \mathrm{H}-8 \mathrm{Ar}) ; \\
8.22(1 \mathrm{H}, \mathrm{t}, \mathrm{H}-6 \mathrm{Ar}) ; 8.48(1 \mathrm{H}, \mathrm{s}, \mathrm{H}-2 \mathrm{Ar})\end{array}$ \\
\hline
\end{tabular}


Continuation Table 2

\begin{tabular}{|c|c|c|}
\hline 1 & 2 & 3 \\
\hline 2.18 & $\begin{array}{l}\text { 1.83-2.17 }\left(4 \mathrm{H}, \mathrm{m}, 3,5-\mathrm{CH}_{2}\right) ; 2.26\left(3 \mathrm{H}, \mathrm{s}, 5^{\prime}-\mathrm{CH}_{3}\right) ; \\
\text { 2.95-3.05 }(2 \mathrm{H}, \mathrm{m}) \text { and } 4.25-4.35\left(2 \mathrm{H}, \mathrm{m}, 2,6-\mathrm{CH}_{2}\right) ; \\
3.81\left(3 \mathrm{H}, \mathrm{s}, 2^{\prime}-\mathrm{OCH}_{3}\right) ; 4.75-4.91(1 \mathrm{H}, \mathrm{m}, 4-\mathrm{CH})\end{array}$ & $\begin{array}{l}6.77\left(1 \mathrm{H}, \mathrm{d}, \mathrm{H}-4^{\prime} \mathrm{Ar}\right) ; 6.83\left(1 \mathrm{H}, \mathrm{d}, \mathrm{H}-3^{\prime} \mathrm{Ar}\right) ; 7.30-7.40(2 \mathrm{H}, \mathrm{m}) \\
\text { and } 7.59\left(1 \mathrm{H}, \mathrm{s}, \mathrm{H}-5,8,6^{\prime} \mathrm{Ar}\right) ; 7.54(1 \mathrm{H}, \mathrm{s}, \mathrm{NH}) ; \\
8.23(1 \mathrm{H}, \mathrm{t}, \mathrm{H}-6 \mathrm{Ar}) ; 8.47(1 \mathrm{H}, \mathrm{s}, \mathrm{H}-2 \mathrm{Ar})\end{array}$ \\
\hline 2.19 & $\begin{array}{l}\text { 1.78-2.11 }\left(4 \mathrm{H}, \mathrm{m}, 3,5-\mathrm{CH}_{2}\right) ; 2.16\left(3 \mathrm{H}, \mathrm{s}, 2^{\prime}-\mathrm{CH}_{3}\right) ; \\
2.28\left(3 \mathrm{H}, \mathrm{s}, 4^{\prime}-\mathrm{CH}_{3}\right) ; 2.90-3.01(2 \mathrm{H}, \mathrm{m}) \text { and } \\
4.25-4.35\left(2 \mathrm{H}, \mathrm{m}, 2,6-\mathrm{CH}_{2}\right) ; 4.75-4.90(1 \mathrm{H}, \mathrm{m}, 4-\mathrm{CH})\end{array}$ & $\begin{array}{l}6.92\left(1 \mathrm{H}, \mathrm{d}, \mathrm{H}-5^{\prime} \mathrm{Ar}\right) ; 6.99\left(1 \mathrm{H}, \mathrm{s}, \mathrm{H}-3^{\prime} \mathrm{Ar}\right) ; 7.08(1 \mathrm{H}, \mathrm{d} \\
\left.\mathrm{H}-6^{\prime} \mathrm{Ar}\right) ; 7.30-7.45(2 \mathrm{H}, \mathrm{m}, \mathrm{H}-5,8 \mathrm{Ar}) ; 7.96(1 \mathrm{H}, \mathrm{s}, \mathrm{NH}) ; \\
8.24(1 \mathrm{H}, \mathrm{t}, \mathrm{H}-6 \mathrm{Ar}) ; 8.49(1 \mathrm{H}, \mathrm{s}, \mathrm{H}-2 \mathrm{Ar})\end{array}$ \\
\hline 2.20 & $\begin{array}{l}\text { 1.80-2.13 }\left(4 \mathrm{H}, \mathrm{m}, 3,5-\mathrm{CH}_{2}\right) ; 2.20\left(3 \mathrm{H}, \mathrm{s}, 2^{\prime}-\mathrm{CH}_{3}\right) ; \\
\text { 2.91-3.04 }(2 \mathrm{H}, \mathrm{m}) \text { and } 4.25-4.38\left(2 \mathrm{H}, \mathrm{m}, 2,6-\mathrm{CH}_{2}\right) ; \\
\text { 4.76-4.93 }(1 \mathrm{H}, \mathrm{m}, 4-\mathrm{CH})\end{array}$ & $\begin{array}{l}7.03\left(1 \mathrm{H}, \mathrm{dd}, \mathrm{H}-4^{\prime} \mathrm{Ar}\right) ; 7.15\left(1 \mathrm{H}, \mathrm{d}, \mathrm{H}-3^{\prime} \mathrm{Ar}\right) ; 7.30-7.42(3 \mathrm{H}, \\
\left.\mathrm{m}, \mathrm{H}-5,8,6^{\prime} \mathrm{Ar}\right) ; 8.08(1 \mathrm{H}, \mathrm{s}, \mathrm{NH}) ; 8.24(1 \mathrm{H}, \mathrm{t}, \mathrm{H}-6 \mathrm{Ar}) ; \\
8.48(1 \mathrm{H}, \mathrm{s}, \mathrm{H}-2 \mathrm{Ar})\end{array}$ \\
\hline 2.21 & $\begin{array}{l}\text { 1.81-2.14 }\left(4 \mathrm{H}, \mathrm{m}, 3,5-\mathrm{CH}_{2}\right) ; 2.90-2.99(2 \mathrm{H}, \mathrm{m}) \text { and } \\
\text { 4.33-4.45 }\left(2 \mathrm{H}, \mathrm{m}, 2,6-\mathrm{CH}_{2}\right) ; 3.74\left(3 \mathrm{H}, \mathrm{s}, 3^{\prime}-\mathrm{OCH}_{3}\right) ; \\
\text { 4.80-4.93 }(1 \mathrm{H}, \mathrm{m}, 4-\mathrm{CH})\end{array}$ & $\begin{array}{l}6.48\left(1 \mathrm{H}, \mathrm{m}, \mathrm{H}-2^{\prime} \mathrm{Ar}\right) ; 7.03-7.20\left(3 \mathrm{H}, \mathrm{m}, \mathrm{H}-4^{\prime}, 5^{\prime}, 6^{\prime} \mathrm{Ar}\right) ; \\
7.64(1 \mathrm{H}, \mathrm{d}, \mathrm{H}-5 \mathrm{Ar}) ; 7.77(1 \mathrm{H}, \mathrm{dd}, \mathrm{H}-7 \mathrm{Ar}) ; 8.10(1 \mathrm{H}, \mathrm{d}, \mathrm{H}-8 \\
\mathrm{Ar}) ; 8.47(1 \mathrm{H}, \mathrm{s}, \mathrm{H}-2 \mathrm{Ar}) ; 8.49(1 \mathrm{H}, \mathrm{s}, \mathrm{NH})\end{array}$ \\
\hline 2.22 & $\begin{array}{l}1.43\left(3 \mathrm{H}, \mathrm{t}, 2^{\prime}-\mathrm{OCH}_{2} \mathrm{CH}_{3}\right) ; 1.75-2.18\left(4 \mathrm{H}, \mathrm{m}, 3,5-\mathrm{CH}_{2}\right) ; \\
2.95-3.10(2 \mathrm{H}, \mathrm{m}) \text { and } 4.20-4.35\left(2 \mathrm{H}, \mathrm{m}, 2,6-\mathrm{CH}_{2}\right) ; \\
4.07\left(2 \mathrm{H}, \mathrm{q}, 2^{\prime}-\mathrm{OCH}_{2} \mathrm{CH}_{3}\right) ; 4.80-4.93(1 \mathrm{H}, \mathrm{m}, 4-\mathrm{CH})\end{array}$ & $\begin{array}{l}\left.\text { 6.80-6.98 (3H, m, H-3, } 4^{\prime}, 5^{\prime} \mathrm{Ar}\right) ; 7.55(1 \mathrm{H}, \mathrm{s}, \mathrm{NH}) ; 7.65(1 \mathrm{H}, \mathrm{d}, \\
\mathrm{H}-5 \mathrm{Ar}) ; 7.75-7.82\left(2 \mathrm{H}, \mathrm{m}, \mathrm{H}-7,6^{\prime} \mathrm{Ar}\right) ; 8.08(1 \mathrm{H}, \mathrm{d}, \mathrm{H}-8 \mathrm{Ar}) ; \\
8.44(1 \mathrm{H}, \mathrm{s}, \mathrm{H}-2 \mathrm{Ar})\end{array}$ \\
\hline 2.23 & $\begin{array}{l}1.75-2.17\left(4 \mathrm{H}, \mathrm{m}, 3,5-\mathrm{CH}_{2}\right) ; 2.83-2.98(2 \mathrm{H}, \mathrm{m}) \text { and } \\
4.30-4.45\left(2 \mathrm{H}, \mathrm{m}, 2,6-\mathrm{CH}_{2}\right) ; 4.10-4.25(4 \mathrm{H}, \mathrm{m}, \\
\left.2 ", 3 \text {, }-\mathrm{CH}_{2}\right) ; 4.78-4.90(1 \mathrm{H}, \mathrm{m}, 4-\mathrm{CH})\end{array}$ & $\begin{array}{l}6.66\left(1 \mathrm{H}, \mathrm{d}, \mathrm{H}-5^{\prime} \mathrm{Ar}\right) ; 6.83\left(1 \mathrm{H}, \mathrm{m}, \mathrm{H}-6^{\prime} \mathrm{Ar}\right) ; 7.05\left(1 \mathrm{H}, \mathrm{s}, \mathrm{H}-2^{\prime}\right. \\
\mathrm{Ar}) ; 7.66(1 \mathrm{H}, \mathrm{d}, \mathrm{H}-5 \mathrm{Ar}) ; 7.78(1 \mathrm{H}, \mathrm{m}, \mathrm{H}-7 \mathrm{Ar}) ; 8.10 \\
(1 \mathrm{H}, \mathrm{d}, \mathrm{H}-8 \mathrm{Ar}) ; 8.34(1 \mathrm{H}, \mathrm{s}, \mathrm{NH}) ; 8.47(1 \mathrm{H}, \mathrm{s}, \mathrm{H}-2 \mathrm{Ar})\end{array}$ \\
\hline 2.24 & $\begin{array}{l}\text { 1.80-2.15 }\left(4 \mathrm{H}, \mathrm{m}, 3,5-\mathrm{CH}_{2}\right) ; 2.95-3.07(2 \mathrm{H}, \mathrm{m}) \text { and } \\
\left.\text { 4.22-4.33(2H, m, 2,6-CH })^{2}\right) ; 3.85\left(3 \mathrm{H}, \mathrm{s}, 2^{\prime}-\mathrm{OCH}_{3}\right) ; \\
\text { 4.77-4.93(1H, m, 4-CH) }\end{array}$ & $\begin{array}{l}6.95(2 \mathrm{H}, \mathrm{s}) \text { and } 7.55-7.85\left(4 \mathrm{H}, \mathrm{m}, \mathrm{H}-5,7,8,3^{\prime}, 4^{\prime}, 6^{\prime} \mathrm{Ar}\right) \\
7.87(1 \mathrm{H}, \mathrm{s}, \mathrm{NH}) ; 8.41(1 \mathrm{H}, \mathrm{s}, \mathrm{H}-2 \mathrm{Ar})\end{array}$ \\
\hline 2.25 & $\begin{array}{l}\text { 1.81-2.17 }\left(4 \mathrm{H}, \mathrm{m}, 3,5-\mathrm{CH}_{2}\right) ; 2.24\left(3 \mathrm{H}, \mathrm{s}, 4^{\prime}-\mathrm{CH}_{3}\right) ; \\
\text { 2.88-2.98 }(2 \mathrm{H}, \mathrm{m}) \text { and } 4.31-4.43\left(2 \mathrm{H}, \mathrm{m}, 2,6-\mathrm{CH}_{2}\right) ; \\
\text { 4.79-4.92 }(1 \mathrm{H}, \mathrm{m}, 4-\mathrm{CH})\end{array}$ & $\begin{array}{l}7.00\left(2 \mathrm{H}, \mathrm{d}, \mathrm{H}-3^{\prime}, 5^{\prime} \mathrm{Ar}\right) ; 7.34\left(2 \mathrm{H}, \mathrm{d}, \mathrm{H}-2^{\prime}, 6^{\prime} \mathrm{Ar}\right) ; 7.51(1 \mathrm{H}, \mathrm{t}, \\
\mathrm{H}-6 \mathrm{Ar}) ; 7.64(1 \mathrm{H}, \mathrm{d}, \mathrm{H}-8 \mathrm{Ar}) ; 7.79(1 \mathrm{H}, \mathrm{t}, \mathrm{H}-7 \mathrm{Ar}) ; \\
8.17(1 \mathrm{H}, \mathrm{d}, \mathrm{H}-5 \mathrm{Ar}) ; 8.44(2 \mathrm{H}, \mathrm{s}, \mathrm{H}-2 \mathrm{Ar}, \mathrm{NH})\end{array}$ \\
\hline
\end{tabular}

The results of screening for the antimicrobial activity of arylamides of 4-(4-oxo-4H-quinazolin-3-yl)piperidine-1-carboxylic acids 2 have shown that they possess not less activity than the reference drugs used in the experiment. The activity of compounds 2.3, 2.12, $2.13\left(\mathrm{X}_{1}=8\right.$-Me, $\mathrm{X}_{2}=3$,4-ethylendioxy; $\mathrm{X}_{1}=8-\mathrm{Cl}, \mathrm{X}_{2}=4$-Et; $\mathrm{X}_{1}=8-\mathrm{Cl}, \mathrm{X}_{2}=3-\mathrm{F}, 4-\mathrm{Me}$, respectively) even exceeded the results of the reference drugs. The high activity was observed mostly against the gram-positive strains, while gram-negative bacteria and fungi were resistant for the compounds tested. It may be applied for construction of the narrow spectrum antibiotics.

The SAR analysis has shown that the presence of substituents in position 8 of the quinazoline cycle and in position 4 of the aromatic fragment of ureas increases their antimicrobial effect against gram-positive bacteria. The common property of all substituents of the most active samples is their positive mesomeric effect, which increases the electron density of the corresponding cycles. Evidently modification of the position 8 of the quinazoline moiety and position 4 of the aromatic cycle of the urea fragment with electron-donating substituents is a good way for design of novel antimicrobials in the series of arylamides of 4-(4-oxo-4H-quinazolin-3-yl)-piperidine-1-carboxylic acids.

\section{Experimental Part}

\section{Chemical Part}

All solvents and reagents were purchased from commercial sources. ${ }^{1} \mathrm{H}$ NMR-spectra were acquired on a Varian Mercury-200 (200 MHz) spectrometer, the solvent was DMSO- $d_{6}$ with TMS as an internal standard. Elemental analysis was performed on an Euro Vector EA-3000 Elemental Analyzer. Melting points were determined on a Kofler bench.

The substituted 3-(piperidin-4-yl)quinazolin$\mathbf{4}(\mathbf{3 H})$-ones (1.1-1.7) were obtained according to the method reported previously [9].

3-(Piperidin-4-yl)quinazolin-4(3H)-one (1.6). M. p. $-179-181^{\circ} \mathrm{C} .{ }^{1} \mathrm{H}$ NMR, $\delta$, ppm: $1.72-2.08(4 \mathrm{H}$, $\left.\mathrm{m}, 3,5-\mathrm{CH}_{2}\right) ; 2.72-3.11(2 \mathrm{H}, \mathrm{m})$ and $4.10-4.23(2 \mathrm{H}, \mathrm{m}$, 2,6- $\left.\mathrm{CH}_{2}\right) ; 4.71-4.92(1 \mathrm{H}, \mathrm{m}, 4-\mathrm{CH}) ; 7.51-7.92(4 \mathrm{H}, \mathrm{m}$, $\mathrm{H}-5,6,7,8 \mathrm{Ar}$ ); 8.45 (1H, s, H-2 Ar). Found, \%: N 17.98. $\mathrm{C}_{13} \mathrm{H}_{15} \mathrm{~N}_{3}$ O. Calculated, \%: N 18.33.

The general method for the synthesis of substituted arylamides of 4-(4-oxo-4H-quinazolin-3-yl)piperidine-1-carboxylic acids (2.1-2.25): Dissolve the corresponding 3-piperidin-4-yl-3H-quinazolin-4-one (1.2 $\mathrm{mmol}$ ) in $10 \mathrm{ml}$ of 2 -propanol at room temperature, then add the corresponding substituted aryl isocyanate $(1.44 \mathrm{mmol})$, and stir the mixture at room temperature for $12 \mathrm{~h}$. After formation of the crystalline precipitate filter it and wash with 2-propanol.

\section{Microbiological Part}

According to the WHO recommendations [15] the following test-strains were used: Staphylococcus aureus ATCC 25923, Escherichia coli ATCC 25922, Pseudomonas aeruginosa ATCC 27853, Bacillus subtilis ATCC 6633, Proteus vulgaris ATCC 4636, Candida albicans ATCC 885/653. The inoculum suspension was prepared using a Densi-La-Meter apparatus (made by 
Table 3

The antimicrobial activity of arylamides of 4-(4-oxo-4H-quinazolin-3-yl)-piperidine-1-carboxylic acids 2

\begin{tabular}{|c|c|c|c|c|c|c|}
\hline \multirow{3}{*}{ No. } & \multicolumn{6}{|c|}{ Diameter of the growth inhibition zones* ${ }^{*} \mathrm{~mm}$} \\
\hline & \multicolumn{3}{|c|}{ Gram-positive bacteria } & \multicolumn{2}{|c|}{ Gram-negative bacteria } & \multirow{2}{*}{$\frac{\text { Fungi }}{\text { C. } a .}$} \\
\hline & S. $a$. & B. C. & E. C. & P. v. & P. $a$. & \\
\hline Metronidazole & 14 & 16 & 14 & 0 & 0 & 14 \\
\hline Synthomycine & 14 & 17 & 17 & 17 & 17 & 0 \\
\hline 2.1 & 14 & 14 & 13 & 0 & 0 & 13 \\
\hline 2.2 & 18 & 18 & 14 & 13 & 12 & 14 \\
\hline 2.3 & 22 & 21 & 15 & 14 & 15 & 15 \\
\hline 2.4 & 12 & 14 & 14 & 12 & 13 & 14 \\
\hline 2.5 & 12 & 13 & 0 & 0 & 0 & 0 \\
\hline 2.6 & 15 & 15 & 14 & 13 & 13 & 13 \\
\hline 2.7 & 15 & 16 & 14 & 13 & 14 & 14 \\
\hline 2.8 & 12 & 14 & 0 & 0 & 0 & 13 \\
\hline 2.9 & 15 & 15 & 13 & 12 & 12 & 13 \\
\hline 2.10 & 14 & 16 & 14 & 12 & 12 & 14 \\
\hline 2.11 & 16 & 16 & 14 & 13 & 13 & 14 \\
\hline 2.12 & 25 & 21 & 16 & 15 & 16 & 15 \\
\hline 2.13 & 23 & 19 & 14 & 14 & 15 & 15 \\
\hline 2.14 & 16 & 16 & 15 & 14 & 14 & 14 \\
\hline 2.15 & 14 & 18 & 14 & 0 & 0 & 16 \\
\hline 2.16 & 12 & 14 & 13 & 0 & 0 & 13 \\
\hline 2.17 & 12 & 14 & 13 & 0 & 0 & 13 \\
\hline 2.18 & 14 & 15 & 15 & 12 & 12 & 15 \\
\hline 2.19 & 14 & 15 & 13 & 13 & 13 & 12 \\
\hline 2.20 & 13 & 17 & 15 & 16 & 15 & 14 \\
\hline 2.21 & 14 & 14 & 13 & 13 & 12 & 14 \\
\hline 2.22 & 15 & 13 & 13 & 13 & 13 & 12 \\
\hline 2.23 & 14 & 14 & 13 & 13 & 12 & 14 \\
\hline 2.24 & 13 & 14 & 13 & 0 & 0 & 13 \\
\hline 2.25 & 16 & 15 & 15 & 14 & 14 & 0 \\
\hline
\end{tabular}

*The average for three experiments.

PLIVA-Lachema, Czech Republic; 540-nm wavelength). The suspension was prepared according to the manual for the device and the information sheet concerning innovation in the healthcare system No. 163-2006 "Standardization for preparation of microbial suspensions", Kyiv. The inoculum density was $10^{7}$ cells in $1 \mathrm{ml}$ of the medium, and it was determined by comparing with McFarland standard [17]. The 18 to 24hour old culture of the microorganism was used for the test. For the antimicrobial study the Mueller-Hinton agar was employed, for the Candida albicans strain the Sabouraud agar was used. The compounds were introduced into agar using the "wells" method [15]. The compounds studied were introduced as $0.3 \mathrm{ml}$ aliquots of DMSO solution (10 mg/ml concentration), the reference drug Metronidazole was used as DMSO solution $(30 \mu \mathrm{g} / \mathrm{ml})$, Syntomycin was used as $\mathrm{H}_{2} \mathrm{O}$ solution $(30 \mu \mathrm{g} / \mathrm{ml})$. The antibacterial activity was assessed by measuring zones of inhibition of the corresponding microorganism.

\section{Conclusions}

The valuable antimicrobial properties of the substituted aryl amides of 4-piperidnyl-1-carboxylic acid and derivatives of 3-(piperidin-4-yl)quinazolin-4(3H)-one inspired us for construction and preparation of the library of the synthetically attractive substituted arylamides of 4-(4-oxo-4H-quinazolin-3-yl)-piperidine1-carboxylic acids obtained by the interaction of 3(piperidin-4-yl)quinazolin-4(3H)-ones with isocyanates in the 2-propanol medium. The results of the antimicrobial activity screening have shown that 
(4-ethylphenyl)amide of 4-(8-chloro-4-oxo-4H-quinazolin-3-yl)piperidin-1-carboxylic acid is more active than the reference drugs Metronidazole and Syntomycin. For the lead compounds the analysis of the structure-activity relationship has evidently shown that modification of position 8 of the quinazoline moiety and position 4 of the aromatic cycle of the urea fragment with electron-donating substituents is a good way for design of novel antimicrobials in the series of arylamides of 4-(4-oxo- $4 \mathrm{H}$ quinazolin-3-yl)-piperidine-1-carboxylic acids.

\section{References}

1. Пат. W02006/117762 (2007). Заявл.: 03.05.2006. Опубл.: 08.02.2007. [Электронный ресурс]. Pежим доступа httр://worldwide.espacenet.com/ publicationDetails/originalDocument?CC=WO\&NR=2006117762A3\&KC=A3\&FT =D\&ND=\&date $=20070208 \& D B=\& \& l o c a l e=e n \_E P$.

2. Пат. US20130090326 (2013). Заявл.: 15.10.2010. Опубл.: 11.04.2013. [Электронный pecyрс]. Pежим доступа http://worldwide.espacenet.com/

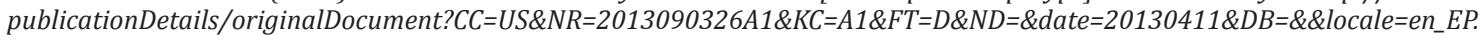

3. Samala G., Devi P. B., Nallangi R., Sridevi J. P., Saxena S., Yogeeswari P., Sriram D. Biorg. Med. Chem., 2014, Vol. 22, No.6, pp.1938-1947.

4. Zheng P., Somersan-Karakaya S., Lu S., Roberts J., Pingle M., Warrier T., Little D., Guo X., Brickner S. J., Nathan C. F. J. Med. Chem., 2014, Vol. 57, No.9, pp. 3755-3772.

5. Пат. W02010103381 (2010). Заявл.: 10.03.2010. Опубл.: 16.09.2010. [Электронный peсурс]. Pежим доступа httр://worldwide.espacenet.com/ publicationDetails/originalDocument?CC $=$ WO\&NR=2010103381A1\&KC $=A 1 \& F T=D \& N D=\&$ date $=20100916 \& D B=\& \&$ locale $=e n_{-} E P$.

6. Christodoulou M. S., Fokialakis N., Passarella D., García-Argáez A. N., Gia O. M., Pongratz I., Dalla Via L., Haroutounian S. A. Biorg. Med. Chem., 2013, Vol. 21, No.14, pp. 4120-4131.

7. Пат. US8691993 (2014). Заявл.: 06.12.2012. Опубл.: 08.04.2014. [Электронный ресурс]. Режим доступа http://worldwide.espacenet.com/ publicationDetails/originalDocument?CC $=U S \& N R=8691993 B 2 \& K C=B 2 \& F T=D \& N D=\&$ date $=20140408 \& D B=\& \& l o c a l e=e n \_E P$.

8. Пат. W02012087938 (2012). Заявл.: 19.12.2011. Опубл.: 28.06.2012. [Электронный ресурс]. Режим доступа https://patentscope.wipo.int/ search/en/detail.jsf?docId=W02012087938.

9. Власов С. В., Кроленко К. Ю., Журавель И. А., Коваленко С. Н. Вестник КазНМУ, 2015, No.3, pр.232-236.

10. Chikkulapalli A., Aavula S. K., Mona N. P. R., Karthikeyan C., Vinodh Kumar C. H., Sulur G. M., Sumathi, S. Tetrahedron Lett., 2015, Vol. 56, No.24, pp.3799-3803.

11. Anandan S.-K., Gless R. D. Bioorg. Med. Chem. Lett., 2010, Vol. 20, No.9, pp.2740-2744.

12. Kołaczek A., Fusiarz I., tawecka J., Branowska D. CHEMIK, 2014, Vol. 68, No.7, pp.620-628.

13. Bhattacharyya S., Pathak U., Mathur S., Vishnoi S., Jain R. RSC Adv., 2014, Vol. 4, No.35, pp.18229-18233.

14. Chaturvedi D. Tetrahedron, 2012, Vol, 68, No.1, pp.15-45.

15. American Society for Microbiology, Coyle M.B. Manual of Antimicrobial Susceptibility Testing. American Society for Microbiology: Washington, 2005, p.236.

16. Clinical and Laboratory Standards Institute. Performance Standards for Antimicrobial Susceptibility Testing; Twenty-Second Informational Supplement. Document M100-S22, 2012, Vol. 32, No.3, CLSI, Wayne, PA, January.

17. McFarland J. Journal of the American Medical Association, 1907, Vol. 49, No.14, pp.1176-1178.

Надійшла до редакції 22.12.2015 р. 